\title{
Carcinoma de células de Merkel: características clínicas y anatomopatológicas, manejo terapéutico y supervivencia en nuestro centro
}

\author{
Merkel cell carcinoma: Clinical and anatomopathological features, management and \\ survival in our centre
}

\author{
Mónica Francés-Monasterio ${ }^{1 *}$, Ángela García-Minarro², Ana B. Paredes-Pérez y Orlando García-Duque ${ }^{1}$ \\ ${ }^{1}$ Servicio de Cirugía Plástica y Reparadora; ${ }^{2}$ Servicio de Dermatología; ${ }^{3}$ Servicio de Anatomía Patológica. Hospital Universitario de Gran Canaria \\ Dr. Negrín, Las Palmas de Gran Canaria, España
}

\begin{abstract}
Resumen
Objetivo: Resumir las características del carcinoma de células de Merkel (CCM) y sistematizar su manejo diagnóstico-terapéutico. Método: Realizamos una búsqueda bibliográfica en PubMed y aparecieron 3,308 artículos, de los que seleccionamos 10 para lectura completa y 22 para lectura del resumen acorde con su contenido. Resultados: En ninguno de nuestros pacientes el CCM fue la primera sospecha diagnóstica. El tratamiento consistió en la extirpación quirúrgica con márgenes libres y linfadenectomía. Se les ofreció radioterapia adyuvante, que rechazaron. Se encuentran libres de enfermedad tras 1 año del tratamiento. Conclusiones: EI CCM es una condición rara y agresiva que se presenta como un nódulo eritematoso de rápido crecimiento y asintomático en zonas fotoexpuestas de pacientes añosos. Los principales factores de riesgo son la exposición ultravioleta, la inmunosupresión y el poliomavirus asociado al carcinoma de Merkel (MCPyV, Merkel cell polyomavirus). La cirugía es el pilar fundamental del tratamiento locorregional. La afectación ganglionar en el transcurso de la enfermedad es uno de los principales factores pronósticos. Si no existen adenopatías reconocibles, debe realizarse biopsia selectiva de ganglio centinela; si existen adenopatías o la biopsia es positiva, está indicada la linfadenectomía. La radioterapia adyuvante está indicada en todos los estadios y ha demostrado un mejor control locorregional. En la enfermedad a distancia es de primera elección la inmunoterapia y participar en ensayos clínicos.
\end{abstract}

Palabras Clave: Carcinoma de células de Merkel. Tratamiento quirúrgico. Características clínicas. Radioterapia.

\begin{abstract}
Objective: To summarize the features of the Merkel cell carcinoma (MCC) and to sistematyze its diagnosis and therapeutic management. Method: We performed a literature review in PubMed, obtaining a total of 3,308 articles, selecting 10 for its complete reading and 22 for the reading of the summary according to the content. Results: In none of our patients, the MCC was the first suspected diagnosis. The treatment consisted in surgical excision with tumor free margins and lymphadenectomy. We offered ad-juvant RT which they rejected. They remain disease-free at the present time. Conclusions: MCC is a rare and aggressive disease which presents as a fast-growing solitary asymptomatic erythematous nodule in those areas of skin which are exposed to sunlight in elderly patients. The main risk factors include radiative ultraviolet, immunosuppression and merkel
\end{abstract}

\footnotetext{
Correspondencia:

*Mónica Francés-Monasterio

Pavía, 21, Bajo izquierda

Fecha de recepción: 24-09-2019

Cir Cir. 2020;88(4):-452

C.P. 35010, Las Palmas de Gran Canaria, España

Fecha de aceptación: 21-01-2020

E-mail: m.francesmon90@outlook.com

DOI: $10.24875 / C I R U .20001596$

Contents available at PubMed

www.cirugiaycirujanos.com

0009-7411/@ 2020 Academia Mexicana de Cirugía. Publicado por Permanyer. Este es un artículo open access bajo la licencia CC BY-NC-ND (http://creativecommons.org/licenses/by-nc-nd/4.0/).
} 
cell polyomavirus. Surgery is the main loco-regional treatment. Lymph node metastases in the course of the disease is one of the main prognostic factors. If there are no adenopaties, sentinel lymph node biopsy must be done; if there are adenopaties or a positive biopsy, lymphadenectomy is indicated. Radiotherapy is indicated in all stages of disease since it has shown to improve loco-regional control. In distant metastatic disease, immunotherapy and participating in clinical trials are the first choice.

Key Words: Merkel cell carcinoma. Surgical management. Clinical features. Radio-therapy.

\section{Introducción}

El carcinoma de células de Merkel (CCM) es un carcinoma cutáneo con diferenciación neuroendocrina con una incidencia en aumento (estimada en 0.7/100,000 personas/año). Su patogénesis está relacionada con el poliomavirus asociado al carcinoma de Merkel (MCPyV, Merkel cell polyomavirus) y con la exposición crónica a la radiación ultravioleta (RUV) ${ }^{1}$. Clínicamente suele presentarse como un nódulo único eritematoso de rápido crecimiento y asintomático en regiones fotoexpuestas de personas de edad avanzada con piel clara, a menudo simulando lesiones benignas. Se caracteriza por su alta recurrencia local y la presencia de metástasis ganglionares, Io que disminuye la supervivencia a los 5 años al 35\%, o al $14 \%$ si existen metástasis a distancia ${ }^{2-4}$. Los avances en el conocimiento de la fisiopatología y la genética molecular de esta enfermedad han permitido ampliar el arsenal terapéutico; sin embargo, la cirugía sigue siendo de primera elección.

\section{Método}

El objetivo del presente artículo es realizar una revisión bibliográfica de lo publicado hasta la fecha acerca del CCM, sus características clínicas y anatomopatológicas, y su manejo terapéutico a raíz de los casos diagnosticados en nuestro centro en el período de enero de 2018 a enero de $2019(n=2)$, con el fin de profundizar los conocimientos de esta afección tan poco frecuente y evaluar el manejo diagnóstico-terapéutico que se ha llevado a cabo. Realizamos una búsqueda bibliográfica en PubMed con las palabras clave Merkel Cell Carcinoma y aparecieron 3308 artículos, de los que seleccionamos 10 para lectura completa y 22 para lectura del resumen acorde con su contenido y fecha de publicación.

\section{Caso 1}

El primer caso es un varón de 87 años, asintomático, con antecedentes de tabaquismo activo, hipertensión

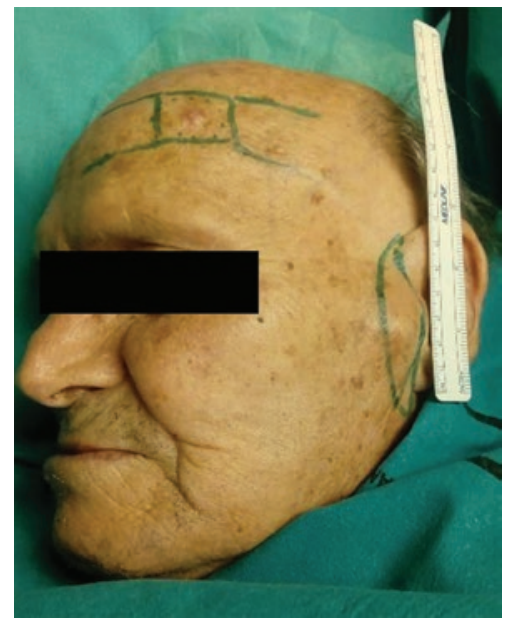

Figura 1. Caso 1: presentación clínica inicial de la lesión frontotemporal y la adenopatía parotídea.

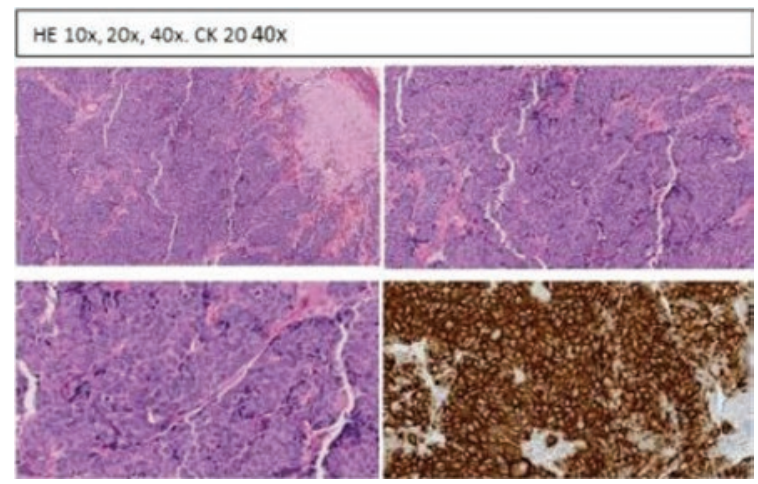

Figura 2. Caso 1: histología de la lesión en la región frontal izquierda.

arterial, dislipidemia, claudicación intermitente e hipertrofia benigna de próstata. Es remitido desde la consulta de dermatología por presentar, en la región frontal izquierda, una lesión rosada pápulo-tumoral de $1.7 \mathrm{~cm}$ de diámetro de 3 meses de evolución, con sospecha clínica de carcinoma basocelular vs. angioqueratoma (Fig. 1). Se realiza una biopsia que arroja el resultado de CCM. En la siguiente revisión se objetiva un nódulo en la región preauricular homolateral, y se realiza punción-aspiración con aguja fina que indica posible metástasis ganglionar intraparotídea. El estudio de extensión con tomografía computarizada no mostró otros hallazgos relevantes, conformando un estadio IIIB (T1N1bM0). Se realizó ampliación de márgenes y 


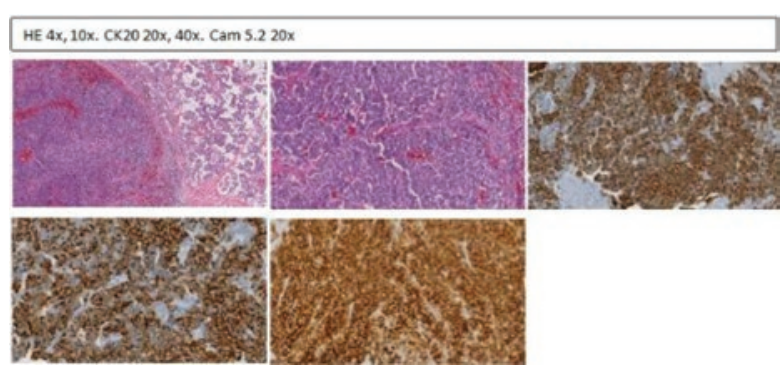

Figura 3. Caso 1: metástasis ganglionar.

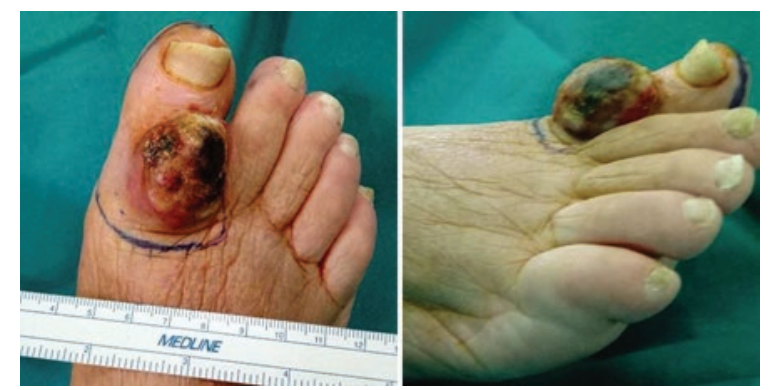

Figura 4. Caso 2: lesión inicial en el dorso del dedo del pie derecho.

parotidectomía superficial sin incidencias, por lo que fue dado de alta. En el análisis anatomopatológico se identificó una lesión de $0.6 \times 0.4 \mathrm{~cm}$, con patrón de crecimiento infiltrativo, que llegaba a la dermis y la hipodermis, respetando los márgenes quirúrgicos de resección. El espesor tumoral medido desde el estrato granuloso hasta el punto de máxima infiltración fue de $4 \mathrm{~mm}$. Respecto al tumour Infiltrating lymphocytes (TIL), se observaba un infiltrado linfocitario difuso peritumoral en la base. Las células presentaban un núcleo redondo, vesicular, con cromatina densa, múltiples nucleolos y un citoplasma escaso. El contaje mitótico fue de 5-6 mitosis/cga, con un índice de proliferación celular Ki67 > 90\%. En cuanto al perfil inmunohistoquímico, expresaba CK20 (en gota), CAM5.2, enolasa, sinaptofisina y neurofilamentos, y era negativo para TTF1 (Fig. 2). La parotidectomía superficial mostró metástasis en un ganglio linfático intraparotídeo con extensión tumoral periganglionar (Fig. 3). A los 12 meses tras el diagnóstico no ha presentado recidivas ni progresión de la enfermedad.

\section{Caso 2}

El segundo caso es una mujer de 78 años con antecedentes de hipertensión arterial, diabetes mellitus tipo 2 de mal control, insuficiencia cardíaca y cardiopatía isquémica estable. Presentaba una lesión tumoral eritematosa de superficie lisa en el dorso del primer dedo del pie derecho, de 6 meses de evolución y asintomática. Fue biopsiada con la sospecha clínica de linfoma vs. melanoma amelanótico (Fig. 4). En un segundo tiempo se realizó extirpación y biopsia selectiva de ganglio centinela (BSGC) inguinal derecho. En los hallazgos anatomopatológicos se observó una tumoración de $3.9 \mathrm{~cm}$, de patrón de crecimiento infiltrativo, con invasión vascular y tumor en margen quirúrgico, compatible con CCM. El espesor tumoral era de al menos $20 \mathrm{~mm}$. La reacción linfocitaria que se apreciaba era escasa y se situaba en todo el componente invasivo del tumor. El resto de las características histológicas e inmunohistoquímicas eran similares a las descritas en el caso previo. Además, se identificó metástasis ganglionar sin extensión extranodal en el ganglio centinela inguinal, conformando finalmente un estadio IIIA (pT4N1aM0). Presentado el caso en el Comité de Tumores, se decidió realizar ampliación de márgenes, que fue negativa para enfermedad residual, y vaciamiento inguinal derecho, que reveló metástasis subcapsular en uno de diez ganglios remitidos (Fig. 5). La paciente fue dada de alta del servicio de cirugía plástica y reparadora a los 3 meses al no presentar complicaciones ni enfermedad residual, para seguimiento por parte de la consulta de dermatología, sin objetivar recurrencia de la enfermedad a los 18 meses tras el diagnóstico, con un Eastern Cooperative Oncology Group (ECOG) de 4 (Fig. 6).

Se ofreció radioterapia adyuvante a ambos pacientes, pero no desearon recibirla.

\section{Discusión}

El diagnóstico clínico del CCM supone un reto por su baja frecuencia y su similitud clínica con otras lesiones, lo que supone que solo se plantee como primera opción diagnóstica en el 1\% de los casos. Se ha propuesto el acrónimo AEIOU (Asintomático, Expansión rápida, Inmunosupresión, mayores de 50 años [Old] y exposición Ultravioleta) ${ }^{5}$ para valorarlo como posibilidad diagnóstica en aquellos casos que reúnan más de tres características.

En la patogénesis del CCM interactúan factores genéticos, moleculares y ambientales. Por un lado, se ha implicado la RUV, basándose en la mayor incidencia en regiones geográficas con niveles más altos de exposición y en su tendencia a aparecer en zonas fotoexpuestas (la mitad aparecen en la cabeza y el cuello) ${ }^{1}$, siendo 25 veces más frecuente en pieles blancas y a menudo hallándose adyacente a otras lesiones asociadas a la RUV, habiéndose objetivado mutaciones de citosina a timina 


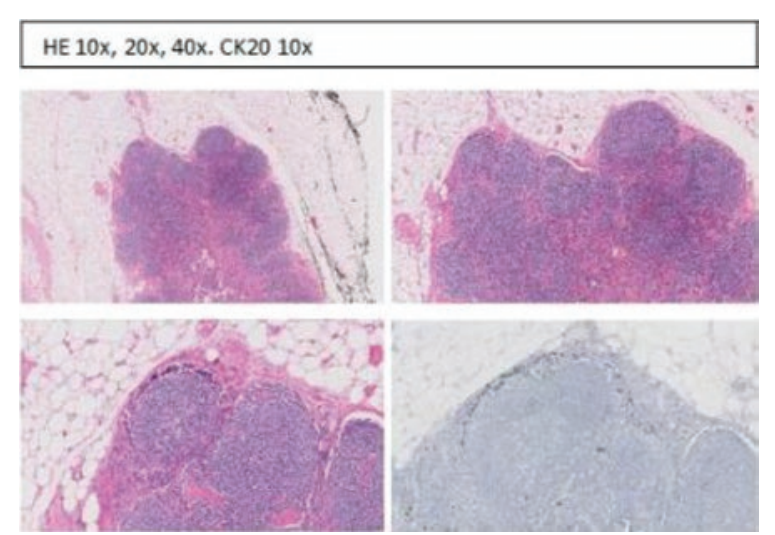

Figura 5. Caso 2: histología de la metástasis inguinal derecha.

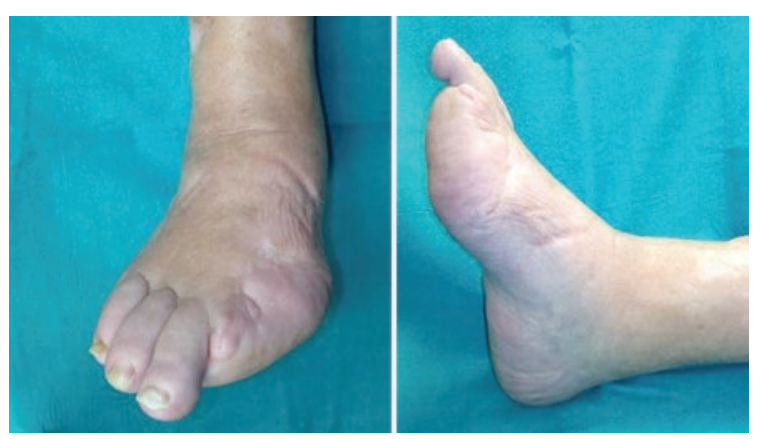

Figura 6. Caso 2: pie derecho a los 3 meses de la cirugía.

(indicativas de daño solar), especialmente en los tumores MCPyV negativos ${ }^{1,6}$.

Un 60 - 97\% de los CCM son MCPyV positivos, quedando caracterizado por primera vez este poliomavirus ubicuo en muestras tumorales de CCM en $2008^{7}$, demostrándose posteriormente su papel en la oncogénesis mediante la integración clonal en el genoma ${ }^{6}$. Asimismo, la inmunosupresión aumenta el riesgo del desarrollo de CCM, y la inmunosenescencia podría explicar el aumento de la incidencia de CCM con la edad 6 .

Debido a la baja incidencia de este tumor y la edad avanzada de los pacientes, no existe un algoritmo terapéutico establecido, pero se acepta que en los estadios I-III el tratamiento debe estar dirigido al tumor primario y al lecho ganglionar regional, siendo de primera elección la cirugía con márgenes amplios (1 - $3 \mathrm{~cm})^{8}$. Debe explorarse el área ganglionar correspondiente en todos los pacientes, si es posible con ecografía. En caso de no presentar adenopatías sospechosas de malignidad, debe realizarse BSGC, ya que hasta en un tercio de estos pacientes se objetivará enfermedad ganglionar microscópica ${ }^{9}$.

La presencia de metástasis ganglionares ocurre en el $30 \%$ de los pacientes en el momento del diagnóstico $^{10}$ y hasta en un $80 \%$ en el transcurso de la enfermedad, siendo el principal predictor de supervivencia, junto con el estadio clínico'. En caso de existir adenopatías clínicamente palpables o radiológicamente sospechosas, deberá realizarse estudio anatomopatológico. El tratamiento de las metástasis ganglionares es la linfadenectomía. En estos casos, está indicado un estudio de extensión.

La radioterapia adyuvante del lecho tumoral está indicada en todos los casos (en especial si hay márgenes afectos, grandes tumores, ganglios clínicamente positivos y factores de mal pronóstico histológico) ${ }^{9}$, ya que el CCM es muy radiosensible. Tras la linfadenectomía se puede plantear radioterapia del territorio de drenaje. En los casos en que sea negativo se recomienda observación debido al bajo porcentaje de falsos negativos de esta técnica ${ }^{8}$. Se acepta que la radioterapia puede disminuir la recidiva locorregional y mejorar la supervivencia global, aunque hasta la fecha no existen estudios aleatorizados que lo demuestren. La radioterapia como modalidad única de tratamiento se realiza en pacientes no candidatos a cirugía?.

Los factores de mal pronóstico incluyen los márgenes quirúrgicos afectos (el más importante de cara a la recurrencia local), la edad superior a 70 años, el sexo masculino, el tamaño superior a $2 \mathrm{~cm}$, la localización en las extremidades y posiblemente los estados de inmunosupresión ${ }^{4,10}$. Por otro lado, los tumores MCPyV positivos presentan un pronóstico más favorable. Los títulos de anticuerpos frente al MCPyV se correlacionan con la carga tumoral y se modifican en respuesta al tratamiento, por lo que se han propuesto para monitorizar la recurrencia ${ }^{11}$, aunque esta determinación no está disponible en nuestro centro. No existen en la actualidad otros marcadores inmunohistoquímicos de mal pronóstico reconocidos.

En cuanto a los pacientes con enfermedad a distancia (estadio IV), se requiere un manejo multidisciplinario. En primera línea se sitúa la inmunoterapia, que incluye los inhibidores de PD-L1 (avelumab) y de PD-1 (pembrolizumab y nivolumab) ${ }^{10}$, que se ha visto en ensayos clínicos no aleatorizados que mejoran las tasas de respuesta mantenida con respecto a la quimioterapia convencional (ciclofosfamida, doxorubicina, vincristina, etopósido, cisplatino), la cual puede ser una alternativa en determinados pacientes (contraindicación de inmunoterapia, etc.). Es preferible la inclusión en un ensayo clínico cuando sea posible ${ }^{10}$. Futuras vías de abordaje terapéutico incluyen los fármacos antivirales, dada la relación con el MCPyV. 
Las recidivas ocurren en los primeros 3 años en el $90 \%$ de los casos, por lo que las últimas guías ${ }^{10}$ recomiendan seguimiento cada 3-6 meses durante los primeros 3 años tras el diagnóstico y posteriormente cada 6 - 12 meses. La mejor prueba para la detección de metástasis es la tomografía por emisión de positrones; por orden de frecuencia, las metástasis afectarán la piel, los pulmones, el hígado, los huesos, el cerebro, la médula ósea y otros órganos ${ }^{12}$.

\section{Conclusiones}

- El CCM es una afección rara y agresiva que se presenta como un nódulo eritematoso de rápido crecimiento y asintomático en zonas fotoexpuestas de pacientes de edad avanzada.

- En su patogenia están implicados la RUV, la inmunosupresión y el MCPyV.

- La cirugía es el pilar fundamental del tratamiento locorregional del CCM.

- Ocho de cada diez pacientes presentarán afectación ganglionar en el transcurso de la enfermedad, siendo uno de los principales factores pronósticos.

- Si no existen adenopatías clínico-radiológicamente reconocibles, debe realizarse BSGC preferiblemente en el mismo acto quirúrgico; si existen adenopatías o la BSGC es positiva, está indicada la linfadenectomía.

- La radioterapia se acepta como adyuvancia en todos los estadios y ha demostrado un mejor control locorregional.

- En la enfermedad a distancia es de primera elección la inmunoterapia y la participación en ensayos clínicos.

\section{Conflicto de intereses}

Los autores declaran no tener conflicto de intereses.

\section{Responsabilidades éticas}

Protección de personas y animales. Los autores declaran que para esta investigación no se han realizado experimentos en seres humanos ni en animales.

Confidencialidad de los datos. Los autores declaran que han seguido los protocolos de su centro de trabajo sobre la publicación de datos de pacientes.

Derecho a la privacidad y consentimiento informado. Los autores han obtenido el consentimiento informado de los pacientes y/o sujetos referidos en el artículo. Este documento obra en poder del autor de correspondencia.

\section{Bibliografía}

1. Becker JC, Stang A, DeCaprio JA. Merkel cell carcinoma. Nat Rev Dis Primers. 201726;3:17077.

2. Harms PW. Update on Merkel cell carcinoma. Clin Lab Med. 2017:37:485-501.

3. Harms KL, Healy MA, Nghiem P, Sober AJ, Johnson TM, Bichakjian CK, et al. Analysis of prognostic factors from 9387 Merkel cell carcinoma cases forms the basis for the new $8^{\text {th }}$ edition AJCC staging system. Ann Surg Oncol. 2016;23:3564-71.

4. Vazquez Doval J, Llombart Cussac B, Pérez Bustillo A, Paradela de la Morena S, Fuente González MJ, Fernández Figueras MT, et al. Diagnosis and treatment of Merkel cell carcinoma in specialized dermatology units: a clinical practice guideline of the Spanish Academy of Dermatology and Venereology. Actas Dermosifiliogr. 2019;110:460-8.

5. Heath M, Jaimes N, Lemos B, Mostaghimi A, Wang LC, Peñas PF, et al. Clinical characteristics of Merkel cell carcinoma at diagnosis in 195 patients: the AEIOU features. J Am Acad Dermatol. 2008;58:375-81.

6. Coggshall K, Tello TL, North JP, Yu SS. Merkel cell carcinoma: an update and review: pathogenesis, diagnosis, and staging. J Am Acad Dermatol. 2018;78:433-42.

7. Feng $\mathrm{H}$, Shuda M, Chang $\mathrm{Y}$, Moore PS. Clonal integration of a polyomavirus in human Merkel cell carcinoma. Science. 2008;319:1096-100.

8. Lemos BD, Storer BE, Iyer JG, Phillips JL, Bichakjian CK, Fang LC, et al. Pathologic nodal evaluation improves prognostic accuracy in Merkel cell carcinoma: analysis of 5823 cases as the basis of the first consensus staging system. J Am Acad Dermatol. 2010;63:751-61.

9. Garbutcheon-Singh KB, Veness MJ. The role of radiotherapy in the management of non-melanoma skin cancer. Australas $\mathrm{J}$ Dermatol. 2019;60:265-72.

10. Merkel Cell Carcinoma, Version 2.2019. NCCN Clinical Practice Guidelines in Oncology. Disponible en: https://merkelcell.org/wp-content/ uploads/2019/01/MCC_v.2.2019.pdf

11. Paulson KG, Lewis CW, Redman MW, Simonson WT, Lisberg A, Ritter D, et al. Viral oncoprotein antibodies as a marker for recurrence of Merkel cell carcinoma: a prospective validation study. Cancer. 2017; 123:1464-74.

12. Medina-Franco H, Urist MM, Fiveash J, Heslin MJ, Bland KI, Beenken SW. Multimodality treatment of Merkel cell carcinoma: case series and literature review of 1024 cases. Ann Surg Oncol. 2001;8:204-8. 